\title{
Video Article \\ Microfluidic Pneumatic Cages: A Novel Approach for In-chip Crystal Trapping, Manipulation and Controlled Chemical Treatment
}

\author{
Afshin Abrishamkar ${ }^{1,2}$, Markos Paradinas ${ }^{3}$, Elena Bailo ${ }^{4}$, Romen Rodriguez-Trujillo ${ }^{5}$, Raphael Pfattner ${ }^{5}$, René M. Rossi ${ }^{1}$, Carmen Ocal ${ }^{5}$, Andrew \\ J. deMello ${ }^{2}$, David B. Amabilino ${ }^{6}$, Josep Puigmartí-Luis ${ }^{1}$ \\ ${ }^{1}$ Empa - Swiss Federal Laboratories for Materials Science and Technology \\ ${ }^{2}$ Institute of Chemical and Bioengineering, Department of Chemistry and Applied Bioscience, ETH Zurich \\ ${ }^{3}$ ICN2-Institut Catala de Nanociencia i Nanotecnologia \\ ${ }^{4}$ WITec GmbH \\ ${ }^{5}$ Institut de Ciència de Materials de Barcelona \\ ${ }^{6}$ School of Chemistry, The University of Nottingham
}

Correspondence to: Josep Puigmartí-Luis at Josep.Puigmarti@empa.ch

URL: https://www.jove.com/video/54193

DOI: doi:10.3791/54193

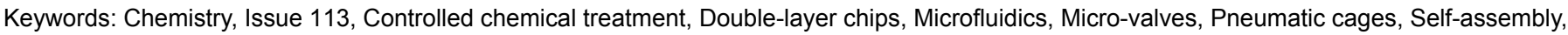
Chemical engineering

Date Published: 7/12/2016

Citation: Abrishamkar, A., Paradinas, M., Bailo, E., Rodriguez-Trujillo, R., Pfattner, R., Rossi, R.M., Ocal, C., deMello, A.J., Amabilino, D.B., Puigmartí-Luis, J. Microfluidic Pneumatic Cages: A Novel Approach for In-chip Crystal Trapping, Manipulation and Controlled Chemical Treatment. J. Vis. Exp. (113), e54193, doi:10.3791/54193 (2016).

\section{Abstract}

The precise localization and controlled chemical treatment of structures on a surface are significant challenges for common laboratory technologies. Herein, we introduce a microfluidic-based technology, employing a double-layer microfluidic device, which can trap and localize in situ and ex situ synthesized structures on microfluidic channel surfaces. Crucially, we show how such a device can be used to conduct controlled chemical reactions onto on-chip trapped structures and we demonstrate how the synthetic pathway of a crystalline molecular material and its positioning inside a microfluidic channel can be precisely modified with this technology. This approach provides new opportunities for the controlled assembly of structures on surface and for their subsequent treatment.

\section{Video Link}

The video component of this article can be found at https://www.jove.com/video/54193/

\section{Introduction}

Molecular materials have long been studied in the scientific community because of their broad number of applications in fields such as molecular electronics, optics and sensors ${ }^{1-4}$. Amongst these, organic conductors are an especially exciting class of molecular materials because of their central role in flexible displays and integrated functional devices ${ }^{5,6}$. However, methodologies used to enable electronic charge transport in molecular-based materials are restricted to the formation of charge transport complexes (CTCs) and charge transport salts (CTSs) ${ }^{7-10}$. Frequently, CTCs and CTSs are generated by electrochemical methods or by direct chemical redox reactions; processes that hamper a controlled transformation of donor or acceptor moieties to more complex architectures where multifunctionality can be conceived. Accordingly, the elucidation of new systematic methods for the controllable generation and manipulation of molecular-based materials remains a significant challenge in the fields of materials science and molecular engineering, and if successful will undoubtedly lead to new functions and novel technological applications.

In this context, microfluidic technologies have recently been used to synthesize molecular-based materials due to their ability to control heat and mass transfer as well as the reaction-diffusion volume of reagents during a synthetic process ${ }^{11,12}$. Put simply, in continuous flows and at low Reynolds numbers a stable interface between two or more reagent streams can be achieved, which affords the formation of a well-controlled reaction zone inside the flow path, where mixing only occurs through diffusion ${ }^{13-16}$. Indeed, we have previously employed laminar flows to localize the synthetic pathway of crystalline molecular materials such as coordination polymers (CPs) inside microfluidic channels ${ }^{17}$. Although this methodology has shown great promise in realizing novel CP nanostructures, the direct integration of such structures onto surfaces, as well as controlled chemical treatment after their formation has yet to be realized in situ ${ }^{18}$. To overcome this limitation, we have recently shown that the actuation of microfluidic pneumatic cages (or valves) incorporated in two-layer microfluidic devices can advantageously be used in this respect. Since the pioneering work of Quake's group ${ }^{19}$, microfluidic pneumatic valves have frequently been used for single-cell trapping and isolation ${ }^{20}$, enzymatic activity investigations ${ }^{21}$, trapping of small fluid volumes ${ }^{22}$, localization of functional materials on surfaces ${ }^{23}$ and protein crystallization ${ }^{24}$ However, we have shown that double layer microfluidic devices can be used to trap, localize and integrate in situ formed structures to read out components and on surfaces ${ }^{18}$. Furthermore, we have also demonstrated that such technology can be used to perform controlled chemical treatments on trapped structures, enabling both, "microfluidic assisted ligand exchange" ${ }^{18}$ and controlled chemical doping of organic crystals ${ }^{18,25}$ In both cases, CTCs could be synthesized under controlled microfluidic conditions, and in the most recent study, multifunctionality could be 
described in the same material piece. Herein, we demonstrate the performance of these double-layer microfluidic devices employing dye-laden flows, generate and control the coordination pathway of a CP as well as its localization on the surface of a microfluidic channel and finally assess controlled chemical treatments onto on-chip trapped structures.

\section{Protocol}

Note: Two layers of a double-layer microfluidic device are designed using a drawing software, e.g., AutoCAD and printed to form high-resolution film masks, with a feature precision limit of $5 \mu \mathrm{m}$. Master molds are created by SU-8 lithography on 4" silicon wafers, allowing the production of structures $50 \mu \mathrm{m}$ in height.

\section{Master Mold Fabrication Using SU-8 Photolithography}

1. Place the silicon wafer on a hot plate set at $200{ }^{\circ} \mathrm{C}$ for $10 \mathrm{~min}$ to dehydrate.

Note: Silicon wafer dehydration provides a better contact and ensures the spreading of the SU-8 photoresist during the spin coating step.

2. Cool the dehydrated wafer down to room temperature over a period of $3 \mathrm{~min}$.

3. Load the wafer onto a spin coater and deposit $4 \mathrm{ml}$ of SU-8 photoresist (approximately $1 \mathrm{ml}$ of SU-8 per inch of substrate) at the center of the wafer.

1. First, spread the deposited SU-8 slowly at 500 revolutions per minute (rpm) for $10 \mathrm{sec}$. Such a rotational speed ensures that SU-8 coverage is increased over the entire wafer surface.

2. Second, control the thickness of the SU-8 by spinning the substrate at higher speeds. In the current experiments, use a spin speed of $3,000 \mathrm{rpm}$ for $30 \mathrm{sec}$ to generate SU-8 features $50 \mu \mathrm{m}$ high.

4. Wipe the edge bead of the wafer carefully with a cotton wipe while spinning at a low rpm (typically $100 \mathrm{rpm}$ ).

5. Heat the spin-coated wafer on a hot plate at $95^{\circ} \mathrm{C}$ for $15 \mathrm{~min}$ to remove residual solvent from the SU-8 (i.e., "soft bake"). Note: The presence of patterns or "wrinkles" in the resist layer indicates the incomplete removal of solvent.

6. Cool the baked wafer back down to room temperature and contact the emulsion printed side of the photomask with the wafer prior to exposure.

7. Turn on the UV-lamp and exposure unit and let the system stabilize over a period of $10 \mathrm{~min}$. Measure the lamp intensity at $365 \mathrm{~nm}$ using an UV-optometer, and estimate the required exposure time (according to time = exposure energy/intensity at $365 \mathrm{~nm}$ ). Note: The exposure energy in the current experiments was calculated to be $250 \mathrm{~mJ} / \mathrm{cm}^{2}$.

8. Expose the photomask on the spin-coated wafer to UV light for the time estimated in the previous step. Here, expose for 79.6 sec.

9. Immediately after exposure, bake the exposed wafer on a hot plate at $65^{\circ} \mathrm{C}$ for $1 \mathrm{~min}$ and subsequently at $95{ }^{\circ} \mathrm{C}$ for $5 \mathrm{~min}$. In this step, the reaction is initiated by UV -light and completed after baking.

10. Leave the post-baked wafer to cool down to room temperature over a period of $3 \mathrm{~min}$.

11. Develop the non-crosslinked SU-8 on the wafer by dissolving it in the SU-8 developer over 8 min. To ensure complete removal of noncrosslinked SU-8, split the process into two steps.

1. In the first, immerse the wafer in the developer solution for $5 \mathrm{~min}$, removing the majority of non-crosslinked SU-8.

2. Then immerse the wafer in a fresh solution of developer for 3 min to dissolve the remaining non-crosslinked SU-8 (typically trapped between crosslinked structures).

12. Rinse the developed wafer with isopropanol and let the wafer having patterned structures (hereinafter referred to as "master mold") stand to dry out. Observation of a milky residue upon rinsing the master mold indicates that development is incomplete.

13. Heat the dried master mold on a hot plate at $200^{\circ} \mathrm{C}$ for $2-5$ min to "hard bake" the substrate and anneal potential cracks in the resist.

14. Allow the fabricated master mold to cool down to room temperature.

15. Place the master mold in a desiccator (connected to a vacuum pump) inside a fume hood.

16. Pour $100 \mu \mathrm{l}$ of trimethylsilyl chloride (TMCS) into a glass beaker and place this inside the desiccator.

CAUTION: TMCS is flammable, corrosive and toxic; thus, handling steps should be performed under a fume hood, with the user wearing protective gloves, goggles and a lab coat.

17. Put the desiccator under vacuum and wait at least $1 \mathrm{hr}$ to allow the TMCS vapor to deposit onto the master mold surface.

18. After $1 \mathrm{hr}$, slowly equilibrate the pressure within desiccator and open to the atmosphere. CAUTION: Do not breathe directly above the open desiccator.

19. Remove the silanized master and close the desiccator.

\section{Fabrication of Double-layer Microfluidic Devices}

Note: The protocol is particularly sensitive to the time and temperature. Any failure to follow to the time frame and temperature may lead to fabrication of non-bonded, and therefore, non-functional devices.

1. Pour a mixture of PDMS elastomer and curing agent (5:1 in weight) into a disposable weighing dish and completely mix with a plastic spatula. In the current experiments, use $50 \mathrm{~g}$ of elastomer and $10 \mathrm{~g}$ of curing agent to form a PDMS layer approximately $5 \mathrm{~mm}$ in height ${ }^{19,26}$

2. Place the well mixed PDMS in a desiccator under vacuum to degas and remove trapped bubbles for $15 \mathrm{~min}$.

3. Mix PDMS elastomer and curing agent (20:1 in weight) in a disposable weighing dish (e.g., $10 \mathrm{~g}$ elastomer and $0.5 \mathrm{~g}$ curing agent) ${ }^{19,26}$.

4. Fix the "control layer" master mold in a frame (in the current experiments, an $11 \mathrm{~mm}$ round polytetrafluoroethylene (PTFE) ring).

5. After $15 \mathrm{~min}$, place the 20:1 PDMS mixture in the desiccator under vacuum for degassing.

6. At the same time as the previous step, take out the 5:1 PDMS mixture from the desiccator and pour this onto the "control layer" master mold that is located inside the round frame. Place the frame containing the PDMS and master mold into the desiccator under vacuum as well. Keep the surplus PDMS. 
7. After $45 \mathrm{~min}$ (and $30 \mathrm{~min}$ after putting both PDMS mixtures into the desiccator), take both PDMS mixtures out of the desiccator and place the frame containing 5:1 PDMS and the "control layer" master mold in an oven at $80^{\circ} \mathrm{C}$.

8. At the same time, begin to spin coat the "fluidic layer" master mold with the 20:1 PDMS mixture. The rotational speed for spin coating is determined based on the desired height, and has been reported elsewhere ${ }^{27}$. Aim to terminate spin coating at 60 min and keep the residual PDMS.

9. After 60 min, put the "fluidic layer" master mold spin coated with $20: 1$ PDMS into an oven at $80^{\circ} \mathrm{C}$.

10. At $75 \mathrm{~min}$, take both master molds out of the oven.

11. Peel off only the 5:1 PDMS from the "control layer" master mold, dice the substrate with a blade and punch the holes for control layers with a $1 \mathrm{~mm}$ biopsy puncher at the inlets positions determined in the design. Here, the control layer is $24 \mathrm{~mm}$ in length and $24 \mathrm{~mm}$ in width.

12. Remove debris from diced chips using adhesive tape.

13. Manually assemble the diced and punched control layer chips on top of the 20:1 PDMS spin coated on the "fluidic layer" master mold using a stereomicroscope with the magnification of $500 \mathrm{X}$ (Figure 1).

14. Pour and draw the residual PDMS around the assembled chips to make a thicker PDMS layer and thereby facilitate removal of the bonded fluidic and control layers at the end.

15. Place the "fluidic layer" master mold containing the two layer devices in an oven at $80^{\circ} \mathrm{C}$ and store overnight.

16. The following day, take the cured assembly out of the oven and allow it to cool down to room temperature.

17. Peel off the PDMS assembly from the "fluidic layer" master mold, dice the fabricated double-layer devices with a blade $(24 \mathrm{~mm}$ in length and $24 \mathrm{~mm}$ in width) and punch fluidic inlets/outlets with a $1.5 \mathrm{~mm}$ biopsy puncher.

18. Treat the surface of chips with open channels and glass coverslips $(24 \mathrm{~mm} \times 40 \mathrm{~mm})$ with a corona discharge and immediately bond them together. Treat by moving the corona discharge over the PDMS slab and glass coverslip over $1 \mathrm{~min}$. Alternatively, use a benchtop plasma system to facilitate bonding.

19. Store the bonded double-layer chips in an oven at $70-80^{\circ} \mathrm{C}$ for at least $4 \mathrm{hr}$.

\section{Microfluidic System Assembly}

1. After the microfluidic device has been assembled, connect the fluidic layer inlets of the chip to the fluidic reservoirs (syringes) using polytetrafluoroethylene (PTFE) tubing $(0.8 \mathrm{~mm}$ id).

2. Connect the pressure supply source to the control layer inlets using silicone rubber tubing and metal connectors that have an external diameter of $1.6 \mathrm{~mm}$.

3. Open and close the valves by applying pressurized air at 3 bar using a pressure source which is operated manually. Supply fluids to the channels using a series of computer controlled syringe pumps.

4. Visualize actuation of valves and device operation with a high resolution camera mounted on an inverted microscope. Use $5 \mathrm{X}$ to $63 \mathrm{X}$ magnification.

\section{Manipulation of the Laminar Flow Regime by Pneumatic Cage Actuation}

Note: The fluidic layer consists of two inlet converging channels, which are $150 \mu \mathrm{m}$ in width, to a wider main channel $300 \mu \mathrm{m}$ in width. And the control layer has a series of identical rectangular valves $(250 \mu \mathrm{m} \times 200 \mu \mathrm{m})$ that are located on top of the main fluidic channel.

1. Once the set-up is connected to the syringe pump and pneumatic controller systems, introduce an aqueous dye flow via one of the inlet channels at the flow rate of $20 \mu \mathrm{l} / \mathrm{min}$.

2. Close the valve by actuating it at 3 bar.

3. Be aware that the fluid can still flow around the valve. This feature is important in achieving controlled chemical treatment of trapped structures $^{18,25}$

4. Open the valve by releasing the pressure.

5. While the dye solution flows through the first channel, inject another aqueous fluid into the second inlet channel at $20 \mu \mathrm{l} / \mathrm{min}$. An interface between two aqueous flows is formed due to the laminar flow regime present in the microfluidic device.

6. Close the valve by actuating it at 3 bar. In this case, the actuation of the valve changes the interface of the two aqueous flows; a result that can be used to modify the synthetic pathway during the formation of a CP (vide infra) ${ }^{18,28}$.

7. Change the fluid flow rates to $30 \mu \mathrm{l} / \mathrm{min}$ and $10 \mu \mathrm{l} / \mathrm{min}$ and observe the down- or up-shifted guiding of the interface generated between the two fluids.

\section{Localization of Microparticles}

1. Connect the fabricated double-layer chip to the syringe pump and pneumatic controller systems.

2. Prepare an aqueous solution containing $10 \mathrm{wt} . \%$ polystyrene fluorescent particles $(5 \mu \mathrm{m}$ in diameter, excitation and emission max at $468 \mathrm{~nm}$ and $508 \mathrm{~nm}$, respectively).

3. Use laser excitation source operating at a wavelength of $488 \mathrm{~nm}$.

4. Introduce the particle-laden fluid into the two inlet channels at a total flow rate of $20 \mu \mathrm{l} / \mathrm{min}$.

5. Wait for 2 min until a stable flow is established.

6. Actuate the valve at 3 bar to close it. Several particles will be trapped underneath the valve and localized on the surface whilst the flow is maintained.

\section{Generation and Controlled Reduction of a Coordination Polymer (CP)}

1. Prepare a $2.5 \mathrm{mM}$ aqueous solution of silver nitrate $\left(\mathrm{AgNO}_{3}\right)$.

2. Prepare a $2.5 \mathrm{mM}$ aqueous solution of cysteine (Cys). 
3. Prepare a saturated ascorbic acid solution in ethanol ${ }^{18}$.

4. Use the same double-layer chip and inject the two reagents into the two inlet channels (one reagent per inlet) each at a flow rate of $50 \mu \mathrm{l} / \mathrm{min}$.

5. Observe the formation of silver and cysteine $(\mathrm{Ag}(\mathrm{I}) \mathrm{Cys}) \mathrm{CPs}$ at the interface of two co-flowing steams.

6. Actuate the valve at 3 bar to trap the formed $\mathrm{Ag}(\mathrm{I}) \mathrm{Cys} \mathrm{CPs}$ underneath the valve.

7. Flush distilled water into the inlet channels at a flow rate of $50 \mu \mathrm{l} / \mathrm{min}$ to wash away the surplus reagents used during the synthetic process.

8. Release the pressure and open the valve. The generated CPs remain underneath the valve under stopped-flow conditions.

9. In order to conduct a controlled chemical reduction of trapped $\mathrm{Ag}(\mathrm{I}) \mathrm{Cys} \mathrm{CPs}$, release the valve pressure at 1 bar and flush the saturated ascorbic acid solution in ethanol at a flow rate of $10 \mu \mathrm{l} / \mathrm{min}$.

10. Observe a clear color change that is attributed to the reduction of $A g(I)$ to metallic silver $(\operatorname{Ag}(0))$ by the ascorbic acid ${ }^{18}$.

\section{Representative Results}

The double layer microfluidic devices consist of two bonded microfluidic chips structured in PDMS as shown in Figure 1. The first layer, which is at the same time bonded to a surface, is used to flow fluids (fluid layer), whilst the second layer, which is directly bonded to the first PDMS layer, is used to flow gas (control layer).

A

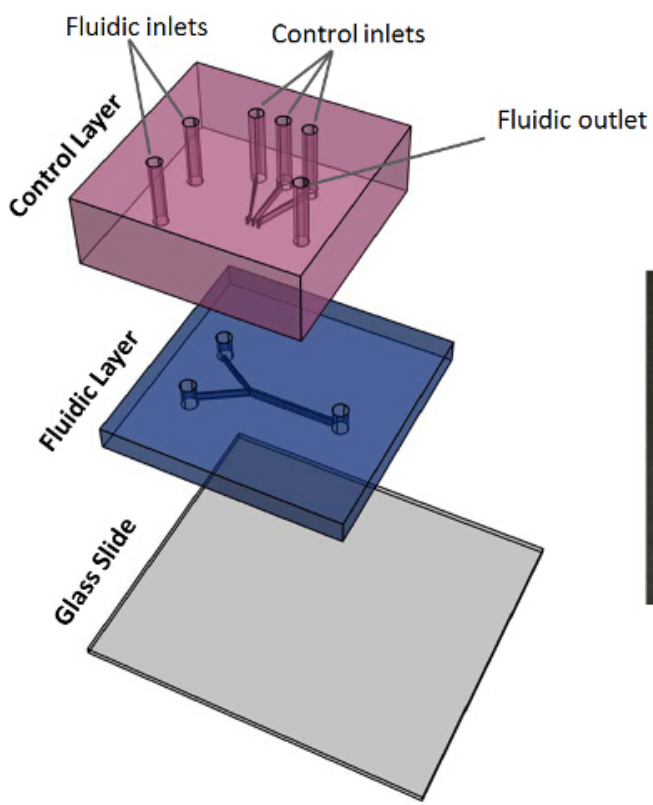

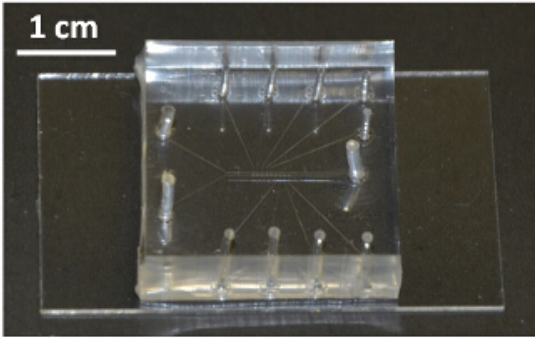

Figure 1. Double-layer microfluidic device. (A) Schematic illustration and (B) micrograph of the double-layer microfluidic device used in our investigations. Please click here to view a larger version of this figure.

Injection of gas through channels in the control layer squeezes the fluid layer towards the surface (Figure 2A and Figure 2B), allowing trapping and localization of structures on the microfluidic channel surface. PDMS membrane actuation can be used to generate pneumatic cages and/or micro-valves that are controlled by a pneumatic controller. As exemplar models of membrane actuation, we show how the complete deflection of the fluid layer avoids a dye-laden flow to circulate underneath the valve after its actuation (Figure 2C) and trapping of fluorescent microparticles on the microchannel surface (Figure 2D and 2E). 
A

Fluidic Layer

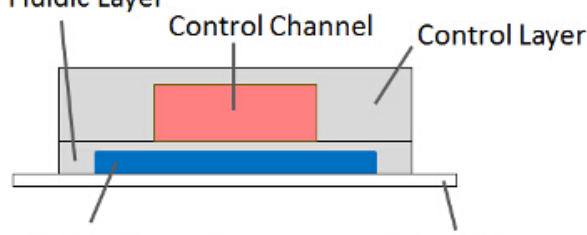

Fluidic Channel

Glass Slide

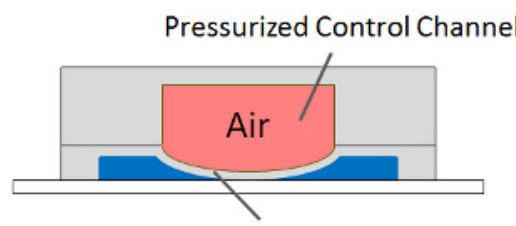

Deflected Membrane

C

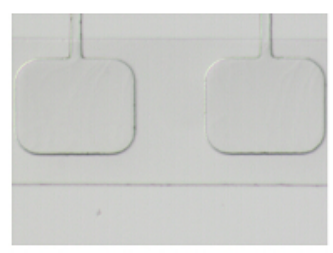

D

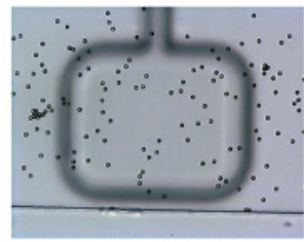

B

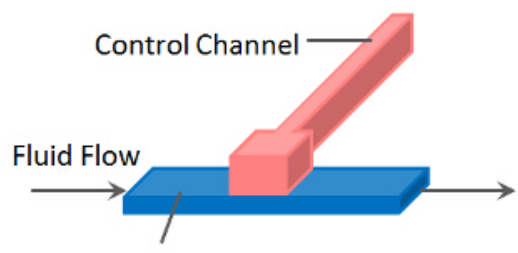

Fluidic Channel

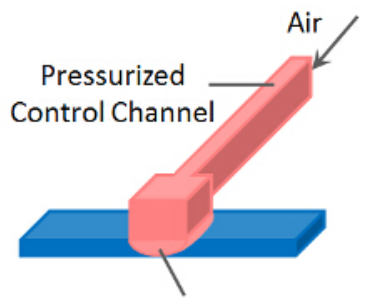

Actuated Valve

E

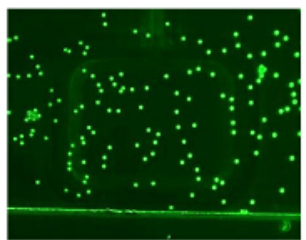

Injection of air

(valves actuated)

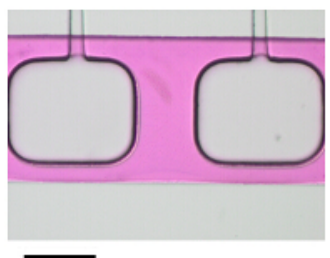

$150 \mu \mathrm{m}$

Injection of air

(valves actuated)

Figure 2. Membrane actuation and trapping of structures. (A) Side and (B) top view illustrations showing the double-layer microfluidic device before (top) and after (bottom) actuation of the pneumatic valve. (C) Micrographs of a double-layer microfluidic device before (top) and after squeezing of the fluid layer (bottom). In bottom panel, the fluid layer is filled with an aqueous solution of rhodamine dye for a better perception of the membrane actuation. (D) Bright-field micrographs of a double-layer microfluidic device before (top) and after (bottom) actuation of the valve with a flowing aqueous solution containing polystyrene fluorescent particles (10 wt.\%). (E) Fluorescent images of the optical microscope images shown in D. Please click here to view a larger version of this figure.

Figure 3A illustrates the trapping of in situ generated CPs inside a double-layer microfluidic device through actuation of a pneumatic cage. Notice that a new coordination pathway is generated after the actuation of the first valve. The valve actuation ensures the trapping of the $\mathrm{Ag}(\mathrm{I})$ Cys $\mathrm{CP}$ generated at the interface of the two reagent streams and facilitates the formation of a new coordination pathway (Figure 3A). A detailed chemical characterization of the $\mathrm{Ag}(\mathrm{I}) \mathrm{Cys} \mathrm{CP}$ generated at the interface of the two reagent streams can be found in previous studies ${ }^{17,18}$ Additionally, and after removal of the surplus reagents solutions with a flow of pure water (Figure 3B), a saturated ascorbic acid solution in ethanol can be added to the microfluidic channel for controlled chemical reduction of on-chip trapped structures (Figure 3C). Reducing the valve pressure from 3 bars to 1 bar favors a controlled chemical treatment of the trapped $\mathrm{Ag}(\mathrm{I}) \mathrm{Cys} \mathrm{CP}$ underneath the clamped area ${ }^{18}$. The color change of trapped $\mathrm{Ag}(\mathrm{I}) \mathrm{Cys} \mathrm{CPs}$ to dark brown is attributed to the reduction of monovalent silver to the metal, in accord with previous observations $^{18,29}$. 


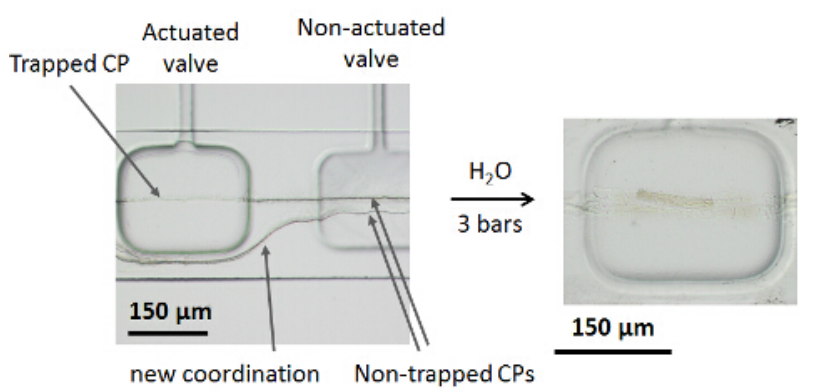

C

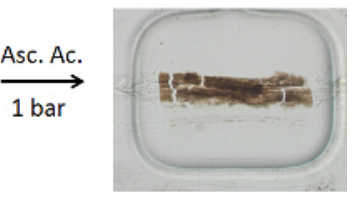

Figure 3. Trapping of $\mathbf{A g}(\mathrm{I}) \mathbf{C y s} \mathrm{CPs}$ and controlled chemical reduction. (A) Optical microscope image showing the trapping of an in situ synthetized $\mathrm{Ag}(\mathrm{I}) \mathrm{Cys} \mathrm{CP}$ and generation of a new coordination pathway. (B) Micrograph of trapped CPs underneath the clamped area after removal of surplus reagents solutions with a water flow, and in (C), micrograph of the same micro-valve after the reduction reaction process. Please click here to view a larger version of this figure.

\section{Discussion}

The reported approach can be easily modified to fabricate different valve shapes to afford other applications such as fluid confinement. Indeed, the flexibility of this protocol also allows for modification of the thickness of the bottom layer, and thereby of the PDMS membrane, from a couple of tens to a few hundreds of microns to fulfill any application of interest. Moreover, dimensions of structures in each layer of the device can be optimized for the desired application and various heights of structures on the master molds can be simply achieved by spinning the photoresist at different velocities. Spinning the photoresist at a higher speed results in thinner structures.

To better implement the protocol, a clean room environment for the fabrication of the master molds is substantially essential; otherwise, the fabrication procedure will lead to defective master molds and thereby to unusable microfluidic devices. Two critical aspects should be emphasized in this protocol: i) the constant temperature of the oven that needs to be adjusted to $80^{\circ} \mathrm{C}$ and ii) the programmed time period between processes that has to be complied accurately. Any modification of temperature and time frame in the protocol might lead to non-bonded chips, and thus, to non-functional devices.

The "turbulent free" conditions typically encountered in microfluidic systems have recently been employed for the generation of microstructures or molecular materials inside ${ }^{30}$ and outside single layer microfluidic chips ${ }^{31}$. In double-layer microfluidic chips, the laminar flow regime, and hence, the interface generated between continuous co-flows can be manipulated using pneumatic cages ${ }^{18,28}$. These devices also provide for effective control over the synthetic pathway, which in turn leads to precise localization and trapping on surfaces ${ }^{18}$.

As mentioned earlier, pneumatic actuation in double-layer microfluidic chips has been previously employed for various applications such as cell trapping ${ }^{20}$, enzymatic activity studies ${ }^{21}$ and protein crystallization ${ }^{24}$. However, the main objective of the reported approach is to propose a platform to be used for trapping and directing the coordination pathway of a crystalline molecular material and controlling chemical reactions onto on-chip trapped structures ${ }^{18,25}$.

The described method does not only allow trapping of anisotropic structures but can be used to localize particles onto surfaces. Future studies can be effectively directed towards the design of new valve shapes for additional application in biology, materials science and sensor technologies. The combination of different valve shapes as well as altered channel heights and membrane thicknesses can be employed to fulfill specific applications, such as chemical studies based on diffusional mixing and the localization of material growth.

A further application of the described microfluidic platforms is in the controlled chemical doping of crystals, which can lead to a rationalized formation of interfaces in crystalline structures ${ }^{19}$. This approach also provides for a wide range of post-treatments of on-chip trapped structures; a methodology that will undoubtedly open new horizons in materials engineering.

It is important to underline that the number of technologies enabling controlled chemical reactions under dynamic conditions and onto crystalline matter are very limited at present, hence making this approach very attractive in materials-related fields. However, a major limitation of this technology is the use of PDMS. PDMS elastomer is incompatible with many organic solvents, which limits the number of reactions that can be conducted inside these microfluidic chips. In future, the development of other elastomers that can tolerate and be stable against a broader number of organic solvents will be highly required in order to expand this field of research to other materials and chemistries.

\section{Disclosures}

The authors have nothing to disclose.

\section{Acknowledgements}

Authors would like to thank the financial support from Swiss National Science Foundation (SNF) through the project no. 200021 160174. 


\section{References}

1. Li, Y., Liu, T., Liu, H., Tian, M. Z., \& Li, Y. Self-assembly of intramolecular charge-transfer compounds into functional molecular systems. Acc. Chem. Res. 47 (4), 1186-1198 (2014).

2. Zhou, P., Shi, R., Yao, J.-f., Sheng, C.-f., \& Li, H. Supramolecular self-assembly of nucleotide-metal coordination complexes: From simple molecules to nanomaterials. Coord. Chem. Rev. 292 107-143 (2015).

3. Goudappagouda, Chithiravel, S., Krishnamoorthy, K., Gosavi, S. W., \& Babu, S. S. Seeded on-surface supramolecular growth for large area conductive donor-acceptor assembly. Chem. Commun. 51 (52), 10439-10442 (2015).

4. Xu, J. L. et al. Self-Assembled Organic Microfibers for Nonlinear Optics. Adv. Mater. 25 (14), 2084-2089 (2013).

5. Min, S. Y. et al. Organic Nanowire Fabrication and Device Applications. Small. 11 (1), 45-62 (2015).

6. Saito, G., \& Yoshida, Y. Development of conductive organic molecular assemblies: Organic metals, superconductors, and exotic functional materials. Bull. Chem. Soc. Jpn. 80 (1), 1-137 (2007).

7. Ono, G., Izuoka, A., Sugawara, T., \& Sugawara, Y. Structure and physical properties of a hydrogen-bonded self-assembled material composed of a carbamoylmethyl substituted TTF derivative. J. Mater. Chem. 8 (8), 1703-1709 (1998).

8. Alves, H., Molinari, A. S., Xie, H. X., \& Morpurgo, A. F. Metallic conduction at organic charge-transfer interfaces. Nat. Mater. 7 (7), $574-580$ (2008).

9. Puigmarti-Luis, J. et al. Rich Phase Behavior in a Supramolecular Conducting Material Derived from an Organogelator. Adv. Funct. Mater. 19 (6), 934-941 (2009).

10. Goetz, K. P. et al. Charge-transfer complexes: new perspectives on an old class of compounds. J. Mater. Chem. C. 2 (17), $3065-3076$ (2014).

11. Wang, Z. L. et al. One-Step Self-Assembly, Alignment, and Patterning of Organic Semiconductor Nanowires by Controlled Evaporation of Confined Microfluids. Angew. Chem. Int. Edit. 50 (12), 2811-2815 (2011).

12. Elvira, K. S., Solvas, X. C. I., Wootton, R. C. R., \& deMello, A. J. The past, present and potential for microfluidic reactor technology in chemical synthesis. Nat. Chem. 5 (11), 905-915 (2013).

13. Kenis, P. J. A., Ismagilov, R. F., \& Whitesides, G. M. Microfabrication inside capillaries using multiphase laminar flow patterning. Science. 285 (5424), 83-85 (1999).

14. Ismagilov, R. F., Stroock, A. D., Kenis, P. J. A., Whitesides, G., \& Stone, H. A. Experimental and theoretical scaling laws for transverse diffusive broadening in two-phase laminar flows in microchannels. Appl. Phys. Lett. 76 (17), 2376-2378 (2000).

15. Atencia, J., \& Beebe, D. J. Controlled microfluidic interfaces. Nature. 437 (7059), 648-655 (2005)

16. Squires, T. M., \& Quake, S. R. Microfluidics: Fluid physics at the nanoliter scale. Rev. Mod. Phys. 77 (3), $977-1026$ (2005).

17. Puigmarti-Luis, J. et al. Coordination Polymer Nanofibers Generated by Microfluidic Synthesis. J. Am. Chem. Soc. 133 (12), $4216-4219$ (2011).

18. Puigmarti-Luis, J. et al. Localized, Stepwise Template Growth of Functional Nanowires from an Amino Acid-Supported Framework in a Microfluidic Chip. ACS. Nano. 8 (1), 818-826 (2014).

19. Unger, M. A., Chou, H. P., Thorsen, T., Scherer, A., \& Quake, S. R. Monolithic microfabricated valves and pumps by multilayer soft lithography. Science. 288 (5463), 113-116 (2000).

20. Kim, T. K., \& Jeong, O. C. Fabrication of a pneumatically driven single-cell trap. Microelectronic Engineering. 88 (8), $1768-1771$ (2011).

21. Kim, S. et al. High-throughput single-molecule optofluidic analysis. Nat Methods. 8 (3), 242-245 (2011).

22. Hsu, C.-H., \& Folch, A. Microfluidic devices with tunable microtopographies. Applied Physics Letters. 86 (2), 023508 (2005).

23. Cvetkovic, B. Z. et al. Confined synthesis and integration of functional materials in sub-nanoliter volumes. ACS Nano. 7 (1), 183-190 (2013).

24. Hansen, C. L., Skordalakes, E., Berger, J. M., \& Quake, S. R. A robust and scalable microfluidic metering method that allows protein crystal growth by free interface diffusion. Proc Natl Acad Sci U S A. 99 (26), 16531-16536 (2002).

25. Puigmarti-Luis, J. et al. Bottom-up on-crystal in-chip formation of a conducting salt and a view of its restructuring: from organic insulator to conducting 'switch' through microfluidic manipulation. Chem. Sci. 6 (6), 3471-3477 (2015).

26. Thorsen, T., Maerkl, S. J., \& Quake, S. R. Microfluidic large-scale integration. Science. 298 (5593), 580-584 (2002).

27. Schneider, F., Draheirn, J., Kamberger, R., \& Wallrabe, U. Process and material properties of polydimethylsiloxane (PDMS) for Optical MEMS. Sensor. Actuat. A-Phys. 151 (2), 95-99 (2009).

28. Kuhn, P., Puigmarti-Luis, J., Imaz, I., Maspoch, D., \& Dittrich, P. S. Controlling the length and location of in situ formed nanowires by means of microfluidic tools. Lab Chip. 11 (4), 753-757 (2011).

29. Ma, Y. et al. Au@Ag core-shell nanocubes with finely tuned and well-controlled sizes, shell thicknesses, and optical properties. ACS Nano. 4 (11), 6725-6734 (2010).

30. Hou, S. et al. A hydrodynamically focused stream as a dynamic template for site-specific electrochemical micropatterning of conducting polymers. Angew. Chem. Int. Edit. 47 (6), 1072-1075 (2008).

31. Thangawng, A. L., Howell, P. B., Richards, J. J., Erickson, J. S., \& Ligler, F. S. A simple sheath-flow microfluidic device for micro/ nanomanufacturing: fabrication of hydrodynamically shaped polymer fibers. Lab. Chip. 9 (21), 3126-3130 (2009). 